\title{
ESTUDIO PRELIMINAR DEL ACEITE DE LOBO MARINO (Arctocephalus australis)
}

\author{
Quiterio Valencia Mécola ${ }^{1}$, Leonardo Sherón \\ R. $^{2}$, Jesús Chipana ${ }^{2}$, Reina Calcino ${ }^{3}$.
}

\author{
R E S U M E N
}

Se extrajo el aceite contenido en el tejido subcutäneo de cinco lobos marinos capturados en diversas épocas, estableciéndose que la temperatura de extracción por fusión humeda es de $100^{\circ} \mathrm{C}$ y el tiempo de 30 minutos.

El rendimiento del aceite con respecto al peso total de los lobos marinos fluctuó entre 3,06 a 10,34\% de aceire y con respecto al peso total del tejido subcutáneo fluctuó entre 49,04 a 61,33\% de aceite, lo cual dependio del periodo de captura.

$\mathrm{Se}$ ha establecido algunas caracteristicas fisicas y quimicas. Entre las primeras, se ha determinado que la densidad promedio es 0,925, el indice de refracción alcanzó un promedio de 1,4789; la viscosidad un promedio de 0,1691 y el color un valor de 14. En cuanto a los parametros químicos se encontraron en los siguientes promedios: Indice de acidez 1,23\%, indice peróxido 6,25 meq/Kg, indice de yodo 182, indice de saponificación 193,84 y las sustancias insaponificables $0,88 \%$.

Además se determinó la composición de los ácidos grasos, de los cuales está compuesto el aceite de lobo marino, determinändose por analisis cromatográfico de gases, que en resumen promedio dio los siguientes resultados: ácidos grasos saturados $22,47 \%$, ácidos grasos monoinsaturados 37,47\%, ácidos grasos poliunsaturados 33,91\% y ácidos grasos no identificados $6,13 \%$.

\section{A B S T R A C T}

Oil from subdermic tissue was extracted; five sea wolves were caught for this purpose in different times. $100^{\circ} \mathrm{C}$ and 30 minutes west established as extraction temperature by wet fusion and extraction time.

Yeild of oil referred to total weight of sea wolves fluctuated between 3.06 and $10.34 \%$ and referred to subdermic tissue between 49.04 and $61.33 \%$ depending upon time of cath.

Some physical and chemical characteristics have been established. Among the first ones are average density 0.925, refraction index 1.4789 (average), viscosity 0.1691 (average) and colons 14 (average). With regards to chemical parameters the following average values were found: acidity index $1.23 \%$, peroxide index $6.25 \mathrm{Meq} / \mathrm{Kgs}$, iodine index 182, saporification index 193.84 and unsaponifiable substances $0.88 \%$.

Fatty acids composition was also determined using gas chromatography techniques and the average results obtained were: saturated fatty acids $22.47 \%$, monounsaturated fatty acids $37.47 \%$, poliun saturated fatty acids $33.91 \%$ and unidentified fatty acids $6.13 \%$. 


\section{I.INTRODUCCIÓN}

Los lobos marinos que habitan en el litoral peruano pertenecen a dos especies: Otaria flavescens u Otäia byronia (lobo chusco o lobo de un pelo) y Arctocephalus australis (lobo fino o lobo de dos pelos) (TOVAR y FUENTES, 1984).

Estas especies, integrantes de un ecosistema particular, constituyen un recurso natural renovable, que explotándolo racionalmente y aprovechándolo en forma integral (piel, grasa, carne, huesos, etc.), podría transformarse en un importante recurso alternativo para la alimentación humana en el futuro, tanto para consumo directo e indirecto.

En estos últimos 20 años se ha dado impulso a las investigaciones acerca de los aceites extraídos de especies marinas, tales como peces, cetáceos, quelonios, llegándose a establecer que muchas especies tienen aceites en cuya composición se encuentran ácidos grasos poliinsaturados, dentro de los cuales están los llamados "esenciales" que no pueden ser sintetizados por los mamíferos, entre ellos el hombre; los cuales cumplen importantes funciones dentro del organismo para su desarrollo armónico (MAYES, 1988). Dentro de estos ácidos grasos se encuentran los pertenecientes al grupo w3.

El descubrimiento del grupo OMEGA-3(AGA-w3), ha permitido que se les investigue, ya que tienen propiedades medicinales (Cáceres, 1991); en especial el ácido eicosapentenoico (EPA) y el docosahexanoico (DHA), los cuales previenen enfermedades cardiovasculares, baja el contenido de los triglicéridos del suero sanguíneo, evita el incremento de colesterol (Stansby, 1967; Stuart, 1980; Nagakura, 1981 y 1983 , Murray y Mayes, 1988; Amadori, 1989; Bjerregaard, 1992; Sprecher, 1992; Dolecek, 1992; Leaf, 1993; Maclennan y Peter, 1993). Además controlan la artritis, dolores menstruales, alergias, jaquecas, envejecimiento prematuro, asma bronquial, potencia sexual; es laxante, humectante(piel), evita la pérdida de cabello (Cáceres, 1991).

Por otro lado, existe relación entre los ácidos grasos (EPA y DHA) y el estre oxidático y la malaria. Se descubrió que tiene una acción antimalárica (Levander, 1992). En la nutrición, los ácidos EPA y $\mathrm{DHA}$, son de trascendental importancia, por cuanto

1. Doctor en Tecnología de Alimentos de Origen Marino.

2. Ingeniero pesquero.

3. Bachiller enQuímica. se tiene que estos se encuentran en la leche materna, siendo la parte grasa fundamental en el organismo humano, por cuanto en los infantes durante esta etapa, forman adecuadamente su tejido nervioso y el cerebro, dando lugar a su funcionamiento ideal en las etapas posteriores (Zaldivar, 1992 y; Leaf, 1993).

Los objetivos centrales del presente trabajo de investigación son los siguientes: optimizar la obtención de aceite crudo de lobo marino; determinar sus características y el contenido de ácidos grasos que lo conforman.

\section{MATERIALES Y MÉTODOS}

\subsection{MATERIA PRIMA}

El material con que se trabajó fue el tejido subcutáneo, extraído de los lobos marinos machos (Arctocephalus australis), cazados en diferentes épocas en Punta Coles, que es el hábitat en la zona sur del Perú y está ubicada en la provincia de llo, departamento de Moquegua. El período de caza fue entre diciembre de 1991 a octubre de 1992. Cada vez que se cazó un ejemplar, inmediatamente se acondicionó en una caja isotérmica, con hielo en escamas, en proporción 1:1 aproximadamente; luego se trasladó hasta el Centro de Producción de Tecnología Pesquera (CEPROTEP) de la Facultad de Ingeniería Pesquera (FAIP) de la Universidad Nacional Jorge Basadre Grohmann de Tacna, donde se sometió a las operaciones siguientes: recepción de materia prima, pesaje, eviscerado, despellejado, separación del tejido subcutáneo y descarnado.

\subsection{TRATAMIENTO DEL TEJIDO SUBCUTÁNEO}

El desarrollo del trabajo fue de acuerdo con el Diagrama № 01. Una vez obtenido el tejido adiposo, se separó los restos de sangre, luego de escurrirlo el material se procedió a cortarlo en cubitos de $2-3 \mathrm{~cm}$, los que se sometieron a congelamiento en tipo IQF para su posterior procesamiento.

\subsection{OPTIMIZACIÓN DEL PROCESO DE EXTRACCIÓN DEL ACEITE}

La separación del aceite se inicia con la molienda, operación que se llevó a cabo en un molino eléctrico de carne, marca APIN de 0,5 HP con criba de $8 \mathrm{~cm}$ de diámetro y orificios de $4 \mathrm{~mm}$ de diámetro. Seguidamente se extrajo el aceite, 
probándose dos métodos, uno por fusión seca y otro por fusión húmeda, lo cual tuvo por finalidad escoger el mejor método de extracción referido a la calidad y cantidad. En ambos métodos, así como en las demás pruebas, se utilizó $0,250 \mathrm{Kg}$ de - muestra cada vez.

\subsubsection{Extracción de aceite por fusión seca.}

Método utilizado por BARRIOS, 1982; el cual consiste en someter en un recipiente al tejido graso y ponerlo bajo la acción del fuego directo, lográndose calentar las paredes y por conducción el tejido adiposo, coagulándose las proteínas y dando a lugar a la liberación de agua y aceite. El agua es eliminada por evaporación, quedando dos productos: el aceite y los residuos de tejido adiposo.

\subsubsection{Extracción de aceite por fusión húmeda}

Método descrito por BAYLE, 1951; BRAUN, 1959; CHEFTEL, 1983 y CALIZAYA, 1988. Se lleva a cabo inmersionando una proporción del tejido adiposo con dos partes de agua caliente a temperaturas y tiempos preestablecidos. La prueba preliminar del presente trabajo se llevó a cabo a una temperatura de $100^{\circ} \mathrm{C}$ y durante 30 minutos.

En los dos casos el aceite se separa mediante un prensado y filtrado, además, en este último por decantación, tanto de la parte acuosa como del presente.

\subsubsection{Optimización de los parámetros de extracción del aceite de lobo marino}

Luego de haber seleccionado el mejor método, se buscó los parámetros más adecuados para la separación del aceite, para lo cual se considera dos variables fundamentales: temperatura y tiempo.

\section{a) Optimización de la temperatura de extracción}

La extracción del aceite se hizo siguiendo el procesamiento descrito anteriormente a temperaturas de $60,70,80,90$ y $100^{\circ} \mathrm{C}$. El tiempo fue de 30 minutos, seleccionándose la temperatura que rindió mayor cantidad de aceite.

\section{b) Optimización del tiempo de extracción}

Obtenida la temperatura óptima $\left(100^{\circ} \mathrm{C}\right)$, el material se procesó bajo estas condiciones variando el tiempo: 15',20', 30', 40' y 50 minutos. Durante el cual deberían coagularse las proteínas lipófilas, dejando en libertad parte del agua y el aceite. Luego del calentamiento, se separaró la fase líquida mediante un prensado, para lo cual cada una de las muestras se introdujeron en un saquillo de algodón, con lo cual se logra obtener la fase líquida, compuesta por agua y aceite, depositándose en recipientes especiales para su decantación, que seguidamente se recogió el aceite obtenido, el cual se depositó en frascos de color ámbar.

\subsection{MÉTODOS ANALÍTICOS}

Con la finalidad de establecer el estado en el cual se encontraba el aceite extraído, se llevaron a cabo los análisis que a continuación se detallan:

\subsubsection{Análisis físico}

\section{a) Densidad}

Determinada mediante el método picnométrico, descrito por PODEZEWSKI, 1980.

\section{b) Indice de Refracción}

Determinado por el método $\mathrm{ABBE}$, descrito por MAIER, 1978; AGENJO, 1980 y MEHLENBACHER, 1979.

\section{c) Viscosidad}

Se determinó por el método descrito por AGENJO(1980), empleando viscosímetro OSTWALD.

\section{d) Color}

Se determinó por el método GARDNER, descrito por MEHLENBACHER, 1979.

\subsubsection{Análisis químico}

\section{a) Indice de acidez}

Se determinó por el método de neutralización con hidróxido de potasio $0,1 \mathrm{~N}$, descrito por ARIMATSU, 1982.

\section{b) Indice de peróxido}

Se determinó por el método LEA modificado, descrito por ARIMATSU, 1982.

\section{c) Indice de yodo}

Fue realizado por el método de HANUS, 
descrito por HARTY FISHER, 1984, que consiste en la determinación del grado de insaturación de los ácidos grasos que componen el aceite.

\section{d) Indice de saponificación}

Se usó el método oficial de la AOAC (Asociation of Official Analytical Chemistry), descrito por HART y FISHER, 1984 y teniendo como referencia lo descrito por BRAUN, 1959.

\section{e) Sustancias insaponificables}

Se utilizó el método de extracción con éter etílico, descrito por BUDSLAWSKI, 1973 y teniendo como referencia a MEHLENBACHER, 1979.

\subsection{DETERMINACIÓN DEL CONTENIDO DE ÁCIDOS GRASOS QUE CONFORMAN EL ACEITE}

Para determinar el contenido de ácidos grasos que están presentes en el aceite de lobo, las diferentes muestras se analizaron en un cromatógrafo de gases líquido, lo cual se logró con el apoyo del Instituto Tecnológico Pesquero del Perú(ITP). Para este proceso se usaron los ésteres metílicos de los ácidos grasos, obtenidos de acuerdo al método METCALFE, 1966, usando el reactivo de trifluoruro de borometanol $\left(\mathrm{BF}_{3}\right.$ al $12,5 \%$ en metanol), y de acuerdo a la norma de ITINTEC N ${ }^{\circ} 209.284$ de setiembre 1985, cuyo título es Preparación de metil ésteres de ácidos grasos. Las condiciones del trabajo y las especificaciones técnicas del equipo fueron las siguientes:

- Cromatógrafo de gases, marca HITACHI, modelo 163.

- Columna de vidrio de $3 \mathrm{~mm}$ de diámetro interno y $2 \mathrm{~m}$ de longitud.

- Material de soporte, $15 \%$ de dietilenglicol succinato DEGS/UNIPORT 8-60-80 de malla.

- Temperatura de la columna (inicial): $160^{\circ} \mathrm{C}$.

- Temperatura del detector (final): $210^{\circ} \mathrm{C}$.

- Programación de temperatura: $1^{\circ} \mathrm{C} / \mathrm{min}$.

- El gas de transporte fue el nitrógeno.

- Flujo de gas: $20 \mathrm{ml} / \mathrm{min}$.

- Estándar interno: octadecano.

- Inyección: 1 microlitro

- Velocidad de carta: $25 \mathrm{~mm} / \mathrm{min}$.

Los metil ésteres de los ácidos grasos fueron identificados por comparación con estándares y tiempo de retención. El porcentaje (peso) de cada ácido graso fue calculado automáticamente por el integrador.

\section{III.RESULTADOS Y DISCUSIÓN}

\subsection{OPTIMIZACIÓN DEL PROCESO DE EXTRACCIÓN DEL ACEITE DE LOBO MARINO}

Los resultados de la extracción de aceite de lobo marino se representan en la Tabla $N^{\circ} 01$ y en el Gráf. $N^{\circ} 01$. En ellas se puede apreciar una notable ventaja en la extracción llevada a cabo por el método de fusión húmeda, lo cual se puede deber en principio, a que los tejidos que envuelven las gotas de grasa está constituido por tejido conectivo, el que está conformado por colágeno y elastina, que forma una red entrecruzada, de tal modo que cuando se hace el proceso de extracción por fusión seca, el calentamiento del recipiente permite que en la parte externa del material rápidamente se desnaturalicen las proteinas, no dejando salir el aceite que se encuentra en el interior, recuperándose la porción que logra salir al exterior. Con el afán de seguir recuperando más, hay necesidad de continuar calentando, con lo cual se logra que vaya cambiando físicamente y quimicamente el aceite, lo cual se pudo apreciar en el cambio de color, el que varía de acuerdo al tiempo que dure el aceite bajo los efectos del calor. La variación del color fue de oro brillante (amarillo intenso) a un color oro viejo (amarillo opaco), según el tiempo. Por otro lado, para determinar la calidad del aceite, se vio por conveniente determinar el valor ácido, teniendo en cuenta que los aceites con el calor excesivo pueden hidrolizarse. Los resultados de los análisis se muestran en la Tabla $N^{\circ} 2$. En la que se puede establecer que aunque se trató de mantener la temperatura constante, en las muestras sometidas a la acción del calor directo, siempre hubo un ligero aumento de la acidez en relación a las muestras extraídas por fusión seca; lo cual puede deberse a que el calentamiento no fue parejo. Las particulas que estuvieron en contacto directo con la superficie directa con el recipiente, recibieron el mayor impacto del calor. En cambio, en las muestras inmersionadas en agua, la temperatura fue homogénea, lográndose calentar incluso más rápido que en el caso anterior. Por las razones expuestas y en base a los resultados mostrados se eligió el método de fusión húmeda para el resto del trabajo. 
3.2 OPTIMIZACIÓN DE LOS PARÁMETROS DE EXTRACCIÓN DEL ACEITE DE LOBO MARINO POR FUSIÓN HÚMEDA

\subsubsection{Optimización de la temperatura de extracción}

Se obtuvieron los resultados que se muestran en la Tabla № 03 y Gráf. № 02 . En ellos podemos observar las temperaturas a las cuales fueron sometidas las muestras, las cantidades de aceite obtenidas en cada proceso con su correspondiente porcentaje. Las muestras para cada temperatura de extracción se hizo por triplicado, resultando que de $250 \mathrm{~g}$ de tejido adiposo tratado a $60^{\circ} \mathrm{C}$, rindió $69 \mathrm{~g}$ de aceite en promedio; sin embargo, la extracción mínima fue de $63 \mathrm{~g}$ y la máxima de $73 \mathrm{~g}$, representando el $25,2 \%$ y el $29,2 \%$, respectivamente, fluctuaciones permisibles para este tipo de trabajo. Con el ascenso de la temperatura, también fue ascendiendo la recuperación de aceite, teniéndose que a $70^{\circ} \mathrm{C}$ se recuperó $82 \mathrm{~g}$ en promedio, lo cual fue un $5 \%$ más que en el caso anterior. La recuperación mínima de aceite a esa temperatura fue de $75 \mathrm{~g}$, lo cual representa el $30 \%$ del peso total de la muestra y la máxima $88 \mathrm{~g}$ que corresponde al $35.2 \%$. Con la prueba realizada a $80^{\circ} \mathrm{C}$ la recuperación de aceite alcanzó a 100 g en promedio, equivalente a $40 \%$ del peso de la muestra, o sea $12 \%$ más a lo obtenido con $60^{\circ} \mathrm{C}$ de temperatura y $7 \%$ más con respecto a la recuperación obtenida trabajando a $70^{\circ} \mathrm{C}$. A la temperatura de $90^{\circ} \mathrm{C}$ la recuperación promedio fue de $115 \mathrm{~g}$, lo cual representa el $46 \%$ de la muestra; la recuperación mínima fue de $113 \mathrm{~g}$ y la máxima de $120 \mathrm{~g}$. Como se puede ver el ritmo del ascenso a esta temperatura es un poco menor que las anteriores, habiendo subido solamente el $6 \%$ con respecto a lo obtenido con una temperatura de $80^{\circ} \mathrm{C}$. En cambio, la diferencia de lo obtenido a 70 y $80^{\circ} \mathrm{C}$ es de $7,2 \%$ $(7,2 \%>6 \%)$. El aceite recuperado a una temperatura de $100^{\circ} \mathrm{C}$ fue $123 \mathrm{~g}$, lo cual representa el $49 \%$ de la muestra total; el aumento del porcentaje fue de $3 \%$, cuyo incremento es mucho menor que el anterior. Como se puede ver en la recuperación de aceite del tejido subcutáneo de lobo marino influye significativamente la temperatura, la cual permite coagular las proteínas del tejido conjuntivo y dejar en libertad el aceite envuelto en estos tejidos, lográndose recuperar en forma óptima, por eso es que se ha decidido establecer como rango óptimo de extracción, las temperaturas comprendidas entre $90^{\circ} \mathrm{C}$ a $100^{\circ} \mathrm{C}$, con lo cual se conseguiría una máxima recuperación, ya que de acuerdo a estudios hechos anteriormente VALENCIA, 1992 se obtiene $67,6 \%$ de aceite, la diferencia de $18,6 \%$ queda adherida al resto del tejido y en el proceso.

\subsubsection{Optimización del tiempo de extracción}

De acuerdo al diseño experimental, uno de los parámetros fundamentales en la extracción de aceite se ha considerado al tiempo de extracción utilizado. Los datos obtenidos se muestran en la Tabla № 04 y Gráf. № 03. En ellos se puede apreciar que el tiempo que dure el proceso influye en la extracción del aceite. Así se tiene que, inmersionando las muestras en agua a temperatura de $100^{\circ} \mathrm{C}$ y manteniéndolas por espacio de 15 minutos, se pudo observar después de realizar el prensado, una recuperación promedio de $85 \mathrm{~g}$ de aceite de $250 \mathrm{~g}$ de muestra, lo cual representa el $34,13 \%$. Paralelamente, con muestras inmersionadas durante 20 minutos se pudo obtener $110 \mathrm{~g}$ o sea $43,87 \%$ de aceite, lo cual viene a ser casi el $10 \%$ de diferencia con respecto a lo anterior y a 30 minutos se obtuvo $123 \mathrm{~g}$ de aceite, lo cual representa el $49 \%$, esto viene a ser aproximadamente el $5 \%$ más que el caso anterior y el $15 \%$ más con respecto al primer caso. Sometiendo las muestras a mayores tiempos de calentamiento se logra un ligero incremento, el cual no es tan significativo; así se tiene que en una inmersión por 40 minutos a $100^{\circ} \mathrm{C}$ se obtuviera $124 \mathrm{~g}$ de aceite en promedio, lo cual representa el $49,53 \%$, o sea un incremento de $0,53 \%$ con respecto al caso anterior y utilizando un tiempo de 50 minutos de inmersión y calentamiento de muestra se obtuvo un ligerísimo incremento en la extracción, o sea de 124 a 125 $\mathrm{g}$, que en porcentaje significa un incremento de únicamente $0,67 \%$. Por estas razones se puede establecer que el tiempo de extracción debe estar entre 30 minutos como máximo.

\subsection{RENDIMIENTO GENERAL DEL ACEITE DE LOS LOBOS MARINOS INVESTIGADOS}

Habiendo seleccionado el proceso de extracción por fusión húmeda como el mejor,y utilizando como parámetros óptimos una temperatura de fusión de $100^{\circ} \mathrm{C}$ por un tiempo 
de 30 minutos, se aplicó al resto de lobos marinos investigados, cuyos resultados se muestran en la Tabla № 05 e ilustrados en el Gráf. № 04.En ellas se puede observar que el peso de los animales fluctuó entre 90,28 a 166,48 Kg, siendo el promedio $111,42 \mathrm{Kg}$. Asimismo, se estableció el contenido de tejido subcutáneo, fluctuó entre 5,30 y $16,85 \mathrm{Kg}$, correspondiendo un rendimiento del contenido subcutáneo con respecto al peso total de 4,99 a $17,20 \%$ y el promedio general fue de $9,91 \%$. De acuerdo al reporte de BARRIOS, 1982 , nos da el $10,8 \%$, el cual es más o menos similar al encontrado en el presente trabajo. Sin embargo, ZAITSEV, 1969 indica que el contenido del tejido subcutáneo de especies de zonas frígidas es de 25 al $46 \%$, esto se debe a que los robos marinos de estas regiones fisiológicamente están adaptados para almacenar gran cantidad de energía, lo cual constituye un elemento de protección. En resumen, las diferentes variaciones del tejido subcutáneo probablemente se debe a diferentes aspectos, tales como: biológicos, distribución geográfica, alimentación, reproducción y estación del año. En la misma tabla se muestra las cantidades de aceite recuperado después de prensado. En la Gráf. № 05 también se muestra la variación de los porcentajes de aceite obtenido del tejido subcutáneo. Como se puede apreciar, tanto en la tabla como en la gráfica, el rango porcentual de variación no es tan grande, el cual es de $10 \%$, por lo que se podría decir que aproximadamente el $55 \%$ del tejido subcutáneo se puede extraer por el método de fusión húmeda y prensado, con lo cual se logra extraer el $81 \%$ del contenido total de aceite y el $19 \%$ restante se queda entre los tejidos conjuntivos. El rendimiento del aceite con respecto al peso total de los lobos marinos investigados, fluctuó entre 3,06 y $10,34 \%$, lo cual parece que depende de la época del año que se captura al animal y el porcentaje de aceite con respecto al peso total del tejido subcutáneo fluctúa entre 49,04 y $61,33 \%$, lo que representa un promedio de $57,20 \%$.

\subsection{CARACTERISTICAS FISICAS DEL ACEITE DE LOBO MARINO FINO}

Los resultados se muestran en la Tabla № 06.

\subsubsection{Densidad}

La densidad fluctúa entre 0,924 y 0,926. Como se puede apreciar, la diferencia es mínima en los aceites de los cinco lobos marinos analizados, pudiéndose afirmar que este aceite tiene en promedio 0,925 de densidad. Si comparamos con los valores de densidad obtenidos por distintos autores en la investigación de aceites de diferentes especies marinas, se puede afirmar que más o menos todos ellos tienen un comportamiento similar, ya que en general el rango de la densidad se encuentra entre $0,917 \mathrm{y}$ 0,935 . La densidad del aceite del lobo marino investigado está dentro de ese rango. Dentro de los mamíferos, el aceite más denso es el de la foca y dentro de los peces, el del atún (ver Tabla № 07). Esto se puede deber fundamentalmente a la distinta composición química de los diferentes aceites.

\subsubsection{INDICE DE REFRACCION}

El índice de refracción a una temperatura de $20^{\circ} \mathrm{C}$ fluctúa entre 1,4779 y 1,4802 , siendo el promedio 1,4789. Este valor se aproxima al mencionado por BARRIOS, 1982; quien encontró que el aceite de lobo marino (Otaria flavescens) chileno tiene un índice de refracción de 1,4811.

\subsubsection{Viscosidad}

Referente a la viscosidad se han encontrado valores que están en un rango entre 0,1567 y 0,1825 , teniendo un valor promedio de 0,1691 expresado en poise. En la bibliografía revisada no se ha encontrado trabajos al respecto, sin embargo COLOM, 1956 dice que 'la viscosidad es una medición esencial para la elección y conocimiento de los aceites para su posterior destino'. Lo único encontrado es el estudio del aceite de la sardina realizado por CALIZAYA, 1988, quien encontró una viscosidad de 0,0673, que comparando con el aceite de lobo marino es más viscoso que el de la sardina.

\subsubsection{Color}

El color del aceite de lobo marino alcanzó valores comprendidos entre 13 y 14 de la escala de Gardner, siendo este último el valor predominante, por lo que se consideró este número como "color del aceite: 14", que comparando con lo obtenido por CALIZAYA, 1988, al analizar el aceite de sardina, obtuvo de 12 a 13 de la misma escala, cuyos valores son aproximados a los que se ha obtenido. 


\subsection{DETERMINACIÓN DE LAS CARACTERÍSTICAS QUÍMICAS DEL ACEITE DE LOBO MARINO}

Los resultados se muestran en la Tabla №08.

\subsubsection{Indice de acidez}

Los valores encontrados para cada uno de los lobos marinos estudiados fueron variados, fluctuando entre 1,03 y $1,30 \%$ de acidez, siendo el promedio general de $1,23 \%$. Estos valores nos indican la variación que tuvo el aceite durante el proceso de extracción, es decir, la separación desde su estado natural en el tejido subcutáneo hasta la obtención del aceite puro, libre de otras sustancias. Para llegar a esto al aceite se le sometió a un proceso térmico, el cual posiblemente permitió fraccionar una pequeña cantidad de aceite. Por otro lado, es posible que haya habido una acción lipolítica (enzimática), lo cual contribuyó al desdoblamiento del aceite. Comparando con los índices de acidez de otras especies marinas, encontrados por diferentes autores, presentados en la Tabla № 09, podemos ver que el aceite de lobo marino de nuestra región se encuentra dentro de los rangos reportados para estas especies; sin embargo, es importante destacar que el índice de acidez del aceite de $O$. flavescens, reportado por CASTILLO, 1989, da un valor de $0,17 \%$, el cual correspondería a un aceite tratado.

\subsubsection{Indice de peróxido}

Los valores del índice de peróxido encontrados están en un rango de 6,05 a 6,5917, siendo el promedio de 6,2517 , los cuales nos indican que la influencia del proceso no fue tan grande, por más que dicho aceite tiene un contenido elevado de ácidos grasos poliinsaturados. El promedio 6,2517 indica los miliequivalentes de peróxido que contiene un $\mathrm{Kg}$ de aceite, lo cual se puede deber a la gran influencia de los catalizadores metálicos, tales como el fierro, provenientes de la sangre y de las proteínas hemáticas; también puede deberse a la acción del proceso, influenciados por muchos factores, tales como: contacto del material con superficies metálicas durante el proceso de molienda, contacto con el aire del medio ambiente del material molido, exposición a la luz y por último al proceso de calentamiento para liberar a las moléculas de aceite. A pesar de estos múltiples factores que pueden haber influido, se puede decir que el oxígeno captado por los triglicéridos es mínimo y en nuestro caso está por debajo del valor indicado para $O$. flavescens y reportado por BARRIOS, 1982; lo cual demostraría un mayor tiempo de almacenamiento del aceite y los resultados del análisis también son menores que los presentados por CALIZAYA, 1988, para el aceite de sardina, que en ese caso podría ser por acción del proceso. También ZALDIVAR, 1989 menciona que los estándares internacionales referentes a la calidad de los aceites para consumo humano, se consideran aptos aquéllos que presentan menos de $10 \mathrm{meq} / \mathrm{Kg}$, lo cual indicaría que nuestro aceite está dentro del rango permisible para el consumo humano, necesitando únicamente un estabilizante para almacenamiento y uso posterior.

\subsubsection{Indice de yodo}

Los resultados que se presentan en la Tabla № 08 nos indican que las muestras investigadas se encuentran en un rango comprendido entre 177,04 y 186,48 , siendo el promedio 182 ; que comparado con otras investigaciones efectuadas en aceite de lobo; es uno de los más altos, indicando la gran presencia de ácidos grasos poliinsaturados que contiene. El índice de yodo para su especie similar chilena, citado por BARRIOS, 1982, es de 164; valor que difiere del nuestro, presumiblemente debido al mayor tiempo de almacenamiento, lo que haya producido una oxidación, no aproximándose a nuestro resultado. Para otros aceites marinos, el indice de yodo es variado, siendo el más bajo el encontrado por BERNARDINI, 1986, en sus investigaciones de aceite de ballena, quien encontró un índice de yodo mínimo de 110. El valor más alto de índice de yodo corresponde al atún, el cual es 195. Para concluir, se puede decir que el aceite de lobo marino estudiado es más alto y su uso tiene que estar sujeto fundamentalmente a su estabilización.

\subsubsection{Indice de saponificación}

Los resultados de las investigaciones se muestran en la Tabla № 08, la cual nos indica que el índice de saponificación está comprendido entre 192,67 y 195,30, siendo el promedio 193,84 y la variación mínima. En comparación con los valores reportados por otros investigadores, se puede afirmar que nuestros resultados son mucho más altos, sin embargo, todos ellos 
reflejan casi el mismo comportamiento, salvo el caso del atún, en el que se observa un índice de saponificación de 160 (ver Tabla № 09).

\subsubsection{Sustancias insaponificables}

Se vio por conveniente determinar las sustancias insaponificables, porque dentro de éstas se encuentran otras de gran importancia y usos, tales como vitaminas ( $A, D, E$ y $K$ ), colorantes, hidrocarburos, etc. Los resultados mostrados en la Tabla № 08 indican que están dentro de los límites comprendidos de 0,8297 a 0,9540\%, siendo el promedio $0,88 \%$. Como podemos ver en comparación con otros aceites marinos (Tabla No. 09), los valores encontrados son bastante elevados, siendo los más altos el de la ballena $(3,7 \%)$, hígado de bacalao $(2,7 \%)$, sardina $(1,25 \%)$.

\subsection{DETERMINACIÓN DEL CONTENIDO DE ÁCIDOS GRASOS QUE CONFORMAN EL ACEITE DE LOBO MARINO (Arctocephalus australis)}

Los resultados se muestran en la Tabla №10, que al observarlas y analizarlas se puede deducir que el aceite de cada uno de los especímenes tuvo el mismo comportamiento, no habiendo distorsiones, encontrándose que el ácido mirístico está en una proporción del $4,5 \%$, ácido palmítico $15,3 \%$, ácido esteárico 2,5\%, ácido palmitoleico $8 \%$, ácido oleico $29 \%$, ácido linoleico $1 \%$, ácido linolénico 2,8\%, ácido eicosapentaenoico 5,7\%, ácido docosapentaenoico $4,1 \%$, ácido docosahexaenoico $20 \%$, ácidos grasos saturados $22 \%$, ácidos grasos monoinsaturados $37,5 \%$ y los ácidos grasos poliinsaturados $33,9 \%$, además, $6,1 \%$ de ácidos grasos no identificados.

En la bibliografía revisada los reportes al respecto son escasos se muestran en la Tabla № 11 , en la que se indica la composición de los ácidos grasos de aceites de algunas especies marinas, según estudios realizados por BARRIOS, 1982 y CASTILLO, 1989 en el lobo marino de un pelo (Otaria flavescens). Comparando las Tablas № 10 y 11 podemos ver que los promedios del contenido de ácidos grasos son más o menos parecidos, con ligeras variaciones no significativas, salvo, en algunos casos, el contenido de ácidos grasos saturados. El presentado por BARRIOS, 1982, es $24,8 \%$ y el encontrado en el presente trabajo, $22,47 \%$; o sea con una diferencia aproximadamente de 2,3\%. Donde sí hay variación es en los ácidos grasos monoinsaturados, principalmente en el análisis realizado por CASTILLO, 1989, de la SGS, donde hay una diferencia significativa de $8 \%$, por cuanto indica que el ácido palmítico tiene un valor de $13,9 \%$, a diferencia de nuestro análisis que arrojó un valor de $8,6 \%$. En el ácido oleico se podría decir que los valores son más o menos similares. Con respecto a los ácidos grasos poliinsaturados, hay diferencias significativas con los análisis efectuados por BARRIOS, ya que en nuestros análisis se encontró $33,91 \%$ y en el del citado autor, $25,9 \%$. La diferencia más saltante es en el contenido de ácido docosahexaenoico, BARRIOS reporta un valor de $11,9 \%$ y en nuestros análisis, $20,3 \%$, como promedio. Ninguno de los autores citados presentan valores para el ácido linolénico, salvo CASTILLO, que da un valor de la sumatoria de ácido linolénico y ácido iecosanoico $\left(\mathrm{C}_{20}: 1\right)$ de $7 \%$. El mismo autor reporta la presencia de ácido iecosadienoico, con un valor de 1,2\%; también reporta la presencia de una mezcla del ácido eicosatetraenoico con el ácido docosaenoico $\left(\mathrm{C}_{22}: 1\right)$, con un valor de $2,8 \%$. Desde el punto de vista fisiológico, los ácidos grasos más importantes son el EPA y el DHA pertenecientes a la serie de Omega-3 (MURRAY y MAYES, 1988). En general, el contenido promedio de estos ácidos fue de $26,1 \%$; BARRIOS da un valor de $18,2 \%$, mientras que CASTILLO, $17,9 \%$, valores que son significativamente menores que el encontrado en el presente trabajo.

Comparando los resultados de los análisis de ácidos grasos del aceite de lobo marino con los de algunas otras especies marinas presentadas en la Tabla №12, se puede deducir que la composición de los ácidos grasos poliinsaturados, por ser los más importantes, son más o menos semejantes a los del lobo marino. El contenido de ácidos grasos poliinsaturados del aceite de especies ícticas pelágicas y de carne roja, tales como anchoveta, caballa, sardina y bonito, difiere con el de especies ícticas de carne blanca (congrio, merluza, etc.). Sin embargo, esto no puede ser regla general, ya que, por ejemplo, el arenque que es de carne roja, tiene relativamente un contenido bajo de ácidos grasos poliinsaturados (21\%) y en el de la anchoveta, el contenido es casi igual al del lobo marino. Otras especies hidrobiológicas importantes son las algas marinas que, según el reporte hecho por MASSON y otros, 1988, presentan un contenido de ácido 
iecosapentanoenoico (EPA) de $27 \%$. Un contenido semejante tiene el aceite de jurel, que es más o menos parecido al resultado del análisis.

\section{CONCLUSIONES}

De la discusión precedente se puede concluir lo siguiente:

1. De los métodos utilizados para el proceso de extracción del aceite de lobo marino, el mejor es el llevado a cabo por fusión húmeda.

2. El rendimiento del tejido subcutáneo con respecto al peso total del animal es $9,91 \%$, el rendimiento del aceite extraido con respecto al peso total del animal es $5,72 \%$ y el rendimiento del aceite con respecto al peso del tejido subcutáneo es $57,2 \%$.

3. Las características físicas del aceite de lobo marino son: densidad 0,925 , índice de refracción 1,4749 , viscosidad 0,1691 y color 14 .

4. Las características quimicas del aceite de lobo marino son: indice de acidez $1,23 \%$, indice de peróxido $6,2517 \mathrm{meq} / \mathrm{Kg}$., indice de yodo 182 , indice de saponificación 193,84 mg/g y sustancias insaponificables $0,88 \%$.

5. El aceite de lobo de mar está constituido por:

TABLA N $N^{\circ} 1$ : Rendimiento comparativo de la extracción del aceite de lobo marino, mediante dos métodos $\left(100^{\circ} \mathrm{C}\right.$ y 30 minutos $)$.

\begin{tabular}{|c|c|c|c|c|c|}
\hline \multirow{2}{*}{$\begin{array}{l}\text { NRO. DE } \\
\text { MUESTRA }\end{array}$} & \multirow{2}{*}{$\begin{array}{l}\text { CANTIDAD DE } \\
\text { MUESTRA }\end{array}$} & \multicolumn{2}{|c|}{ EXTRACCION POR FUSION SECA } & \multicolumn{2}{|c|}{ EXTRACCION POR FUSION HUMEDA } \\
\hline & & $\begin{array}{l}\text { Acete obterido } \\
\text { en } \mathrm{Kg} \text {. }\end{array}$ & $\%$ & $\begin{array}{l}\text { Acete obtenido } \\
\text { en } \mathrm{Kg}\end{array}$ & $\%$ \\
\hline 1 & 0,250 & 0.073 & 29,20 & 0,112 & 44,80 \\
\hline 2 & 0,250 & 0,083 & 32,00 & 0,120 & 48,00 \\
\hline 3 & 0,250 & 0,075 & 30,00 & 0,102 & 40,80 \\
\hline 4 & 0,250 & 0,076 & 30,40 & 0,105 & 42,00 \\
\hline PROMEDIO & 0,250 & 0,076 & 30,40 & 0,110 & 43.90 \\
\hline
\end{tabular}

TABLA N² 2: Variación del valor ácido (Indice de acidez por el tipo de proceso utilizado.

\begin{tabular}{|c|c|c|}
\hline \multirow{2}{*}{$\begin{array}{c}\text { NRO. DE } \\
\text { MUESTRA }\end{array}$} & \multicolumn{2}{|c|}{ PORCENTAJE DE ACIDEZ DEL ACEITE DE LOBO MARINO } \\
\cline { 2 - 3 } & EXTRACCION POR FUSION SECA & EXTRACCION POR FUSION HUMEDA \\
\hline 1 & 1,85 & 1,03 \\
2 & 2,02 & 1,20 \\
3 & 1,79 & 0.79 \\
4 & 4,59 & 1,18 \\
\hline PROMEDIO & 1,80 & 1,05 \\
\hline
\end{tabular}

$22,47 \%$ de ácidos saturados, $37,47 \%$ de ácidos grasos monoinsaturados, $33,91 \%$ de ácidos grasos poliinsaturados y $6,3 \%$ de ácidos no identificados. De los ácidos grasos poliinsaturados, $5,71 \%$ corresponde al EPA, el $4,10 \%$ al DPA y el $20,30 \%$ al DHA.

\section{RECOMENDACIONES}

Teniendo en cuenta la importancia de las caracteristicas y la composición del aceite de lobo marino fino (Arctocephalus australis), se recomienda lo siguiente:

1. Seguir con los estudios de estabilización del aceite, para su uso posterior, tal como lo indican BARRIOS, 1982 y CACERES, 1991.

2. Continuar con los estudios del comportamiento del aceite y establecer sus posibles usos farmacológicos e industriales.

3. Realizar estudios sobre la obtención de ácidos grasos individuales, especialmente el EPA y DHA. Actualmente existe un enorme interés en el mundo científico por estos dos ácidos grasos poliinsaturados, por tener propiedades medicinales y fisiológicas, y que son relativamente abundantes en el aceite de lobo marino.

TABLA N $N^{\circ} 3$ : Obtención del aceite de lobo marino, de acuerdo a la temperatura utilizada en el proceso de optimización.

\begin{tabular}{|c|c|c|c|c|c|c|}
\hline \multirow{2}{*}{ PRUEBA } & \multirow{2}{*}{$\begin{array}{c}\text { TEJIDO SUB } \\
\text { CUTANEO Kg }\end{array}$} & $\begin{array}{c}60^{\circ} \mathrm{C} \\
\mathrm{kg}\end{array}$ & $\begin{array}{c}70^{\circ} \mathrm{C} \\
\mathrm{kg}\end{array}$ & $\begin{array}{c}80^{\circ} \mathrm{C} \\
\mathrm{kg}\end{array}$ & $\begin{array}{c}90^{\circ} \mathrm{C} \\
\mathrm{kg}\end{array}$ & $\begin{array}{c}100^{\circ} \mathrm{C} \\
\mathrm{kg}\end{array}$ \\
\hline 1 & 0,250 & 0,073 & 0,083 & 0,100 & 0,120 & 0,123 \\
2 & 0,250 & 0,063 & 0,075 & 0,105 & 0,113 & 0.120 \\
3 & 0,250 & 0,070 & 0,088 & 0,095 & 0,113 & 0.125 \\
\hline PROMEDIO & 0,250 & 0,069 & 0,082 & 0,100 & 0,115 & 0,123 \\
\hline
\end{tabular}

TABLA N $N^{\circ} 4$ : Obtención del aceite de lobo marino, de acuerdo al tiempo empleado en el proceso de optimización a $100^{\circ} \mathrm{C}$.

\begin{tabular}{|c|c|c|c|c|c|c|c|c|c|c|c|}
\hline \multirow{3}{*}{$\begin{array}{c}\text { NRO } \\
\text { DE } \\
\text { MUEST. }\end{array}$} & \multirow{3}{*}{$\begin{array}{l}\text { CANTIDAD } \\
\rightarrow \mathrm{Kg}^{\circ}\end{array}$} & \multicolumn{10}{|c|}{ TIEMPO DE EXTRACCION (MIN) } \\
\hline & & \multicolumn{2}{|c|}{15} & \multicolumn{2}{|c|}{20} & \multicolumn{2}{|c|}{30} & \multicolumn{2}{|c|}{40} & \multicolumn{2}{|c|}{50} \\
\hline & & CANT. & $\%$ & CANT. & $*$ & CANT & $\%$ & CANT & $\%$ & CANT. & $\%$ \\
\hline 1 & 0,250 & 0,082 & 32,8 & 0,110 & 44,0 & 0,123 & 49,0 & 0,125 & 50,0 & 0,125 & 50,0 \\
\hline 2 & 0,250 & 0,090 & 36,0 & 0,105 & 42,0 & 0,120 & 48,0 & 0,123 & 49,0 & 0,124 & 49,0 \\
\hline 3 & 0.250 & 0,084 & 33,6 & 0,114 & 45,6 & 0,125 & 50,0 & 0,124 & 49,6 & 0,125 & 50,0 \\
\hline ME. & 0,250 & 0,085 & 34,13 & 0,110 & 43,87 & 0,123 & 49,0 & 0,124 & 49,53 & 0,125 & 49,7 \\
\hline
\end{tabular}


TABLA N $N^{\circ}$ : Rendimientos de la recuperación de aceite de lobos marinos (Arctocephalus australis) capturados durante diversas épocas.

\begin{tabular}{|l|c|c|c|c|c|c|}
\hline $\begin{array}{l}\text { PERIODO } \\
\text { DE } \\
\text { CAPTURA }\end{array}$ & $\begin{array}{c}\text { PESO TOTAL } \\
\text { DEL LOBO } \\
(\mathrm{Kg})\end{array}$ & \multicolumn{2}{|c|}{ TEJIDO SUBCUTANEO } & \multicolumn{3}{|c|}{ ACEITE OBTENIDO } \\
\cline { 3 - 7 } & PESO (Kg) & $\begin{array}{c}\text { \% TEJIDO } \\
\text { SUBCUTANEO }\end{array}$ & $\begin{array}{c}\text { CANTIDAD } \\
\text { (Kg) }\end{array}$ & $\begin{array}{c}\text { \% RESPECTO } \\
\text { PESO TOTAL }\end{array}$ & $\begin{array}{c}\text { \% RESPECTO } \\
\text { TEJ. SUBCUT }\end{array}$ \\
\hline DIC. 91 & 112,46 & 10,46 & 9,30 & 5,13 & 4,56 & 49,04 \\
FEB. 92 & 89,93 & 5,30 & 5,89 & 2,89 & 3,21 & 54,53 \\
JUL. 92 & 166,48 & 8,30 & 4,99 & 5,09 & 3,06 & 61,33 \\
SET. 92 & 90,28 & 11,00 & 12,18 & 6,71 & 7,43 & 61,00 \\
OCT. 92 & 97,94 & 16,85 & 17,20 & 10,13 & 10,34 & 60,12 \\
\hline PROMEDIO & 111,42 & 10,38 & 9,91 & 5,99 & 5,72 & 57,20 \\
\hline
\end{tabular}

TABLA $N^{\circ} 6$ : Promedio de los análisis de algunas características físicas del aceite de lobo marino a $20^{\circ} \mathrm{C}$.

\begin{tabular}{|c|c|c|c|c|}
\hline \multirow{2}{*}{ ESPECIMEN } & \multicolumn{4}{|c|}{ CARAC TERIS TICAS FISICAS } \\
\cline { 2 - 5 } & DENSIDAD & $\begin{array}{c}\text { INDICE DE } \\
\text { REFRACCION }\end{array}$ & VISCOSIDAD & $\begin{array}{c}\text { COLOR } \\
\text { (ESC. GARDNER) }\end{array}$ \\
\hline II & 0,926 & 1,4793 & 0,1643 & 13 \\
III & 0,925 & 1,4778 & 0,1752 & 13 \\
IV & 0,926 & 1,4802 & 0,1664 & 14 \\
V & 0,925 & 1,4779 & 0,1567 & 14 \\
\hline PROMEDIO & 0,924 & 1,4795 & 0,1829 & 14 \\
\hline
\end{tabular}

TABLA $N^{\circ} 7$ : Comparación de las caracteristicas físicas del aceite de lobo marino con otras especies marinas a $20^{\circ} \mathrm{C}$ (Según autores).

\begin{tabular}{|c|c|c|c|c|}
\hline \multirow{2}{*}{ ESPECIMEN } & \multicolumn{4}{|c|}{ CARACTERISTICAS FISICAS } \\
\hline & DENSIDAD & $\begin{array}{c}\text { INDICE DE } \\
\text { REFRACCION }\end{array}$ & VISCCSIDAD & \begin{tabular}{|c|c} 
COLOR \\
(ESC. GARDNER) \\
\end{tabular} \\
\hline LOBO EN ESTUDIO & $0,924-0,926$ & $1,4778-14802$ & $0,1567-0,1820$ & $13-14$ \\
\hline BALLENA & $0,917^{(2)} \cdot 0,927$ & $1,470^{(1)}-1,477$ & - & $\cdots$ \\
\hline FOCA & $0,920^{(2)}-0,935$ & $1,468^{(2)}-1,474$ & - & + \\
\hline LOBO MARINO & - & $\begin{array}{c}(3) \\
1,4811\end{array}$ & $\ldots$ & - \\
\hline SARDINA & $0,929-0,934$ & $1,476-1,485$ & $0,0600^{(4)}-0,0673$ & $12^{(4)}-13$ \\
\hline ARENQUE & $0,920-0,936$ & $1,466-1,472$ & - & - \\
\hline ATUN & $0,929^{(1)}-0,935$ & $1,486-1,487$ & - & H \\
\hline $\begin{array}{l}\text { HIGADO DE } \\
\text { BACALAO }\end{array}$ & $0,922^{(1)}-0,932$ & $1,474-1,478$ & - & $\ldots$ \\
\hline
\end{tabular}

(1) Bernardini, 1986; (2) Braun, 1959; (3) Barrios, 1982: (4) Calizaya, 1988
TABLA $N^{\circ} 8$ : Promedios de algunas características químicas del aceite crudo de lobo marino.

\begin{tabular}{|c|c|c|c|c|c|}
\hline \multirow{2}{*}{ ESPECIMEN } & \multicolumn{5}{|c|}{ CAR A C TER I T ICAS } \\
\cline { 2 - 6 } & $\begin{array}{c}\text { INDICE DE } \\
\text { ACIDEZ }\end{array}$ & $\begin{array}{c}\text { INDICE DE } \\
\text { PEROXIDO }\end{array}$ & $\begin{array}{c}\text { INDICE DE } \\
\text { YODO }\end{array}$ & $\begin{array}{c}\text { INDICE DE } \\
\text { SAPONIFICAC. }\end{array}$ & $\begin{array}{c}\text { SUSTANCIAS } \\
\text { INSAPONIFIC }\end{array}$ \\
\hline I & 2,29 & 6,0968 & 177,04 & 192,93 & 0,8297 \\
II & 1,28 & 6,5917 & 183,53 & 193,49 & 0,8515 \\
III & 1,03 & 6,0500 & 186,48 & 192,67 & 0,8738 \\
IV & 1,30 & 6,0700 & 184,50 & 195,30 & 0,9540 \\
V & 1,25 & 6,4512 & 178,45 & 194,80 & 0,8910 \\
\hline PROMEDIO & 1,27 & 6,2517 & 182,00 & 193,84 & 0,8800 \\
\hline
\end{tabular}

TABLA $N^{\circ} 9$ : Comparación de las características químicas del aceite de lobo marino con otras especies marinas.

\begin{tabular}{|c|c|c|c|c|c|}
\hline \multirow[b]{2}{*}{ ESPECIES } & \multicolumn{3}{|c|}{ CARACTERISTICAS } & \multicolumn{2}{|l|}{ QUIMICAS } \\
\hline & $\begin{array}{l}\text { INDICE DE } \\
\text { ACIDEZ (\%) }\end{array}$ & $\begin{array}{l}\text { INDICE DE } \\
\text { PEROXIDO }\end{array}$ & $\begin{array}{l}\text { INDICE DE } \\
\text { YODO }\end{array}$ & $\begin{array}{c}\text { INDICE DE } \\
\text { SAPONIFICAC }\end{array}$ & $\begin{array}{l}\text { SUSTANCIAS } \\
\text { INSAPONIFIC }\end{array}$ \\
\hline LOBO EN ESTUDIO & $1,03-1,30$ & - & $178-186$ & $192-195$ & $0,83-0,95$ \\
\hline BALLENA & $\cdots$ & - & $110-135$ & $185-194$ & $0,7^{(2)}-3,7$ \\
\hline FOCA & $1-20$ & - & $130-152$ & $189^{(1)}-196$ & $0,5^{(2)}-1,0$ \\
\hline LOBO MARINO & $\begin{array}{l}(6) \\
0,17\end{array}$ & $\begin{array}{c}(5) \\
14,3\end{array}$ & $\begin{array}{l}(1) \\
164\end{array}$ & $\begin{array}{c}\text { (1) } \\
1924\end{array}$ & (5) \\
\hline SARDINA & $1,50^{(5)}-2,0$ & $15-20$ & $170-193$ & $189-193$ & $0,75^{(5)}-1,25$ \\
\hline ARENQUE & $2,60-5,7$ & - & $124-128$ & $179-194$ & $0,75^{(5)}-1,3$ \\
\hline ATUN & $\cdots$ & - & $160-195$ & $160-180$ & $\ldots$ \\
\hline $\begin{array}{l}\text { HIGADO DE } \\
\text { BACALAO }\end{array}$ & - & - & $135-168$ & $171^{(2)}-193$ & $\begin{array}{l}\text { (2) } \\
2,7\end{array}$ \\
\hline
\end{tabular}

(1) Bernardini, 1986; (2) Braun, 1959; (3) Bayle, 1951; (4) Barrios, 1982; (5) Calzaya, 1988; (6) Castilo, 1989.

TABLA N $N^{\circ} 10$ : Contenido de ácidos grasos en el aceite de lobo marino (Arctocephalus australis).

\begin{tabular}{|l|r|r|r|r|r|r|}
\hline \multicolumn{1}{|c|}{$\begin{array}{c}\text { ACIDOS } \\
\text { GRASOS }\end{array}$} & \multicolumn{5}{|c|}{ C A R A C T E R I S T I C A S } & Q U I M I C A S \\
\cline { 2 - 7 } & $\begin{array}{c}\text { DICIEMBRE } \\
1991\end{array}$ & $\begin{array}{r}\text { FEBRERO } \\
1992\end{array}$ & $\begin{array}{c}\text { JULIO } \\
1992\end{array}$ & $\begin{array}{c}\text { SETIEMBRE } \\
1992\end{array}$ & $\begin{array}{c}\text { OCTRUBRE } \\
1992\end{array}$ & PROMEDIO \\
\hline Miristico C14:0 & 4,93 & 4,15 & 4,15 & 4,90 & 4,41 & 4,51 \\
Palmitico C16:0 & 15,14 & 15,83 & 15,83 & 14,70 & 15,40 & 15,38 \\
Estearico C18:0 & 2,05 & 2,95 & 2,95 & 2,80 & 2,70 & 2,69 \\
Palmitoleico C16:1 & 8,81 & 7,96 & 7,96 & 7,80 & 7,90 & 8,09 \\
Oleico C18:1 & 29,05 & 29,05 & 29,77 & 28,90 & 30,39 & 29,43 \\
Linoleico C18:2 & 0,96 & 1,03 & 1,02 & 1,00 & 1,02 & 1,01 \\
Linoleico C18:3 & 2,90 & 2,93 & 2,92 & 2,11 & 3,10 & 2,79 \\
EPA C20.5 & 5,60 & 5,65 & 5,75 & 5,75 & 5,82 & 5,71 \\
DPA C22.5 & 4,08 & 3,72 & 4,20 & 4,90 & 5,58 & 4,10 \\
DHA C22.6 & 20,79 & 19,97 & 20,42 & 21,50 & 18,83 & 20,30 \\
\% AC. saturados & 22,12 & 22,93 & 22,39 & 22,40 & 22,51 & 22,47 \\
\% AC. moncisaturados & 37,86 & 37,01 & 37,60 & 37,70 & 38,29 & 37,49 \\
\% AC. Polinsaturados & 34,33 & 33,30 & 34,31 & 35,26 & 32,35 & 33,91 \\
\% Ac. no identficado & 5,69 & 6,76 & 5,70 & 6,64 & 6,85 & 6,13 \\
\hline
\end{tabular}


TABLA N $N^{\circ} 11$ : Contenido en ácidos grasos en el aceite de lobo marino (Otaria flavescens), según autores.

\begin{tabular}{|c|c|c|}
\hline \multirow{2}{*}{ ACIDOS GRASOS } & \multicolumn{2}{|c|}{$\%$ RELATIVO } \\
\hline & BARRIOS, 1992 & CASTILLO, 1989 \\
\hline ACIDOS GRASOS SATURADOS & & \\
\hline C12:0 Ac Laurico & Trazas & 0,1 \\
\hline C14:0 Ac. Miristico & 5,0 & 63 \\
\hline C15:0 Ac. Decapentanoico & 0,4 & 0,4 \\
\hline C16:0 Ac. Palimitico & 15,2 & 11,4 \\
\hline C17.0 Ac. Decaheptanoico & 1,7 & 1,2 \\
\hline C18:0 Ac. Esteario & 2,50 & 1,7 \\
\hline C20.0 Ac. Elicosanoico & 0,0 & 0,1 \\
\hline TOTAL ACIDOS GRASOS SATURADOS & 24,8 & 21,1 \\
\hline ACIDOS GRASOS MONOINSATURADOS & & \\
\hline C16:1 Ac. Palmitoleico & 0,0 & 13,9 \\
\hline C18:1 Ac. Oleico & 33,6 & 31,5 \\
\hline C20:01 Ac. Eicosaenolico & 4,5 & 0,0 \\
\hline TOTALACIDOS GRASOSMONOINSATURADOS & 38,1 & 45,4 \\
\hline ACIDOS GRASOS POLIINSATURADOS & & \\
\hline C14:2 Ac. Miristoleico & 0,7 & 0,0 \\
\hline C18:2 AC. Linoleico & 1,0 & 0,2 \\
\hline C18:3 Ac. Linoleico & 0,0 & 0,0 \\
\hline $\mathrm{C} 18: 3+\mathrm{C} 20: 1$ & 0,0 & 7,0 \\
\hline C20:2 Ac. Eicodadienoico & 0,0 & 1,2 \\
\hline C20.3 Ac. Eicosatrienoico & Trazas & 0,0 \\
\hline C20.4 Ac. Eicosatetraenoico & 0,0 & 0,0 \\
\hline $\mathrm{C} 20: 4+\mathrm{C} 221$ Ac. Erucico & 0,0 & 2,8 \\
\hline C20:5 Ac. Eicosapentanoico & 6,3 & 6,4 \\
\hline C20.4 Ac. Docosatetraenoico & 0.0 & Trazas \\
\hline C20.5 Ac. Docosapentaenoico & 6,0 & 4,5 \\
\hline C20.6 Ac. Dosahexaenoico & 11,9 & 11,2 \\
\hline TOTAL ACIDOS GRASOS POLIINSATURADOS & 25,9 & 33,2 \\
\hline NO IDENTIFICADOS & 11,2 & 2,0 \\
\hline$E P A+D H A$ & 18,2 & 17,6 \\
\hline
\end{tabular}

TABLA $N^{\circ} 12$ : Contenido de ácidos grasos en el aceite de algunas especies marinas (\%).

\begin{tabular}{|c|c|c|c|c|c|c|c|c|c|c|c|}
\hline $\begin{array}{c}0 \text { cos } \\
\text { asos }\end{array}$ & $\mid \begin{array}{c}\text { BONATO } \\
\text { (1) }\end{array}$ & VGRIO & $\begin{array}{l}\text { DUREL } \\
\text { (1) }\end{array}$ & $\begin{array}{l}\text { MERLUZZA } \\
\text { (1) }\end{array}$ & inen & $\underset{(4)}{C \operatorname{CrBN} L A}$ & (4) & 5) & sil(2) & ${ }_{(3)}^{A L G A} \cdot$ & ALGA - \\
\hline C14:0 & 7,0 & 5.5 & 2, & 1,6 & 8,2 & 4,7 & 8. & 6,0 & 19,2 & 3,8 & 5,4 \\
\hline C16:0 & 17,0 & 17,8 & 33,0 & 23,2 & 19,1 & 19,62 & 17,0 & 15,0 & 13,4 & 17.9 & 17,5 \\
\hline C18:0 & 2,0 & 3,3 & 13,7 & 2,4 & 3,6 & 4,0 & 4,0 & 2 & 1,2 & 0.5 & 5,9 \\
\hline 16:1 & 12,0 & 9,7 & 4,4 & 8,5 & 10,4 & 7,49 & 10,0 & 0,0 & 7,5 & 21,2 & 23,9 \\
\hline C18:1 & 21,0 & 30,0 & 12,3 & 32,4 & 14,8 & 19,28 & 12,0 & 7,5 & 8,0 & 6,0 & 4,9 \\
\hline C15.2 & 1,0 & 2. & 2, & 0,9 & 1,5 & 1.01 & 4,0 & 0,0 & 1,2 & 2,5 & 3.8 \\
\hline 4 & 2,0 & & & & 3,4 & 0. & 0. & 0,0 & 9,4 & 0,0 & 0,0 \\
\hline $\mathrm{C} 20.1$ & 1.0 & 2. & 1. & 1.3 & 2,3 & 0,00 & 0,0 & 0,0 & 8.1 & 0,0 & 0,0 \\
\hline C20:5 & 22,0 & 8, & 4 & 12,8 & 22,1 & 16,37 & 16,0 & 0,0 & 13,5 & 27,1 & 16,5 \\
\hline & 2,0 & 3 & 0, & & 2,0 & 0,00 & 0,0 & 21,0 & 0,5 & 0,0 & 0.0 \\
\hline $\mathrm{C} 22.6$ & 5,0 & 7.8 & 20,5 & 7,8 & 4,3 & 13,38 & 14,0 & 0.0 & 0,6 & 0,0 & 1,3 \\
\hline
\end{tabular}

(1) Perú Pesquero - IPEMIN Na 3,1990, (2) Masson y otros, 1988; (3) Navarrete, 1989; (4) Zalvidar, 1992; (5) Markovits A. et al, 1989; (6) Zaitsev, 1977

Nannochloropis sp.

Phaeodactylum tricornutum
GRÁFICO $N^{\circ}$ 1: Porcentaje de aceite obtenido por fusión seca y húmeda, según número de muestra.

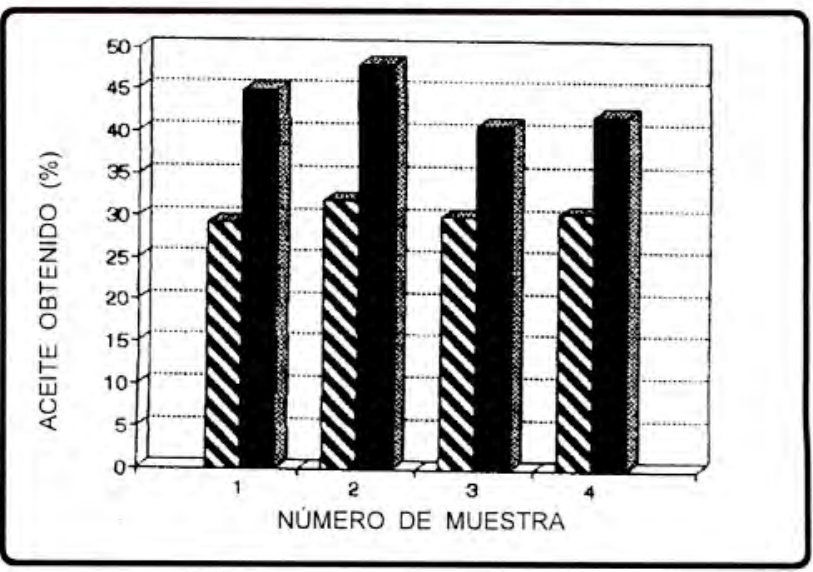

GRÁFICO $N^{\circ}$ 2: Porcentaje de aceite obtenido en fusión a la temperatura de extracción.

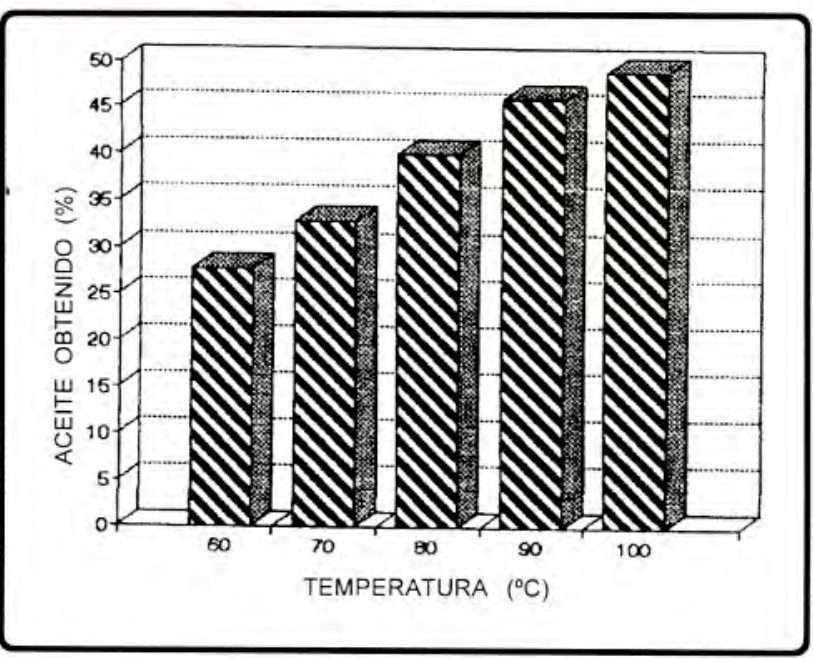

GRÁFICO $N^{\circ} 3$ : Porcentaje de aceite obtenido en función al tiempo de extracción a $100^{\circ} \mathrm{C}$.

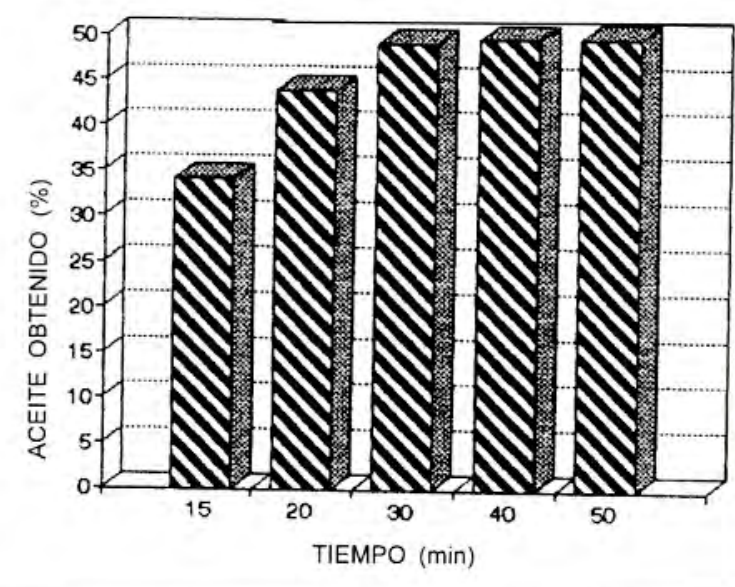


GRÁFICO N 4: Porcentaje del contenido de tejido subcutáneo de acuerdo al periodo de caza.

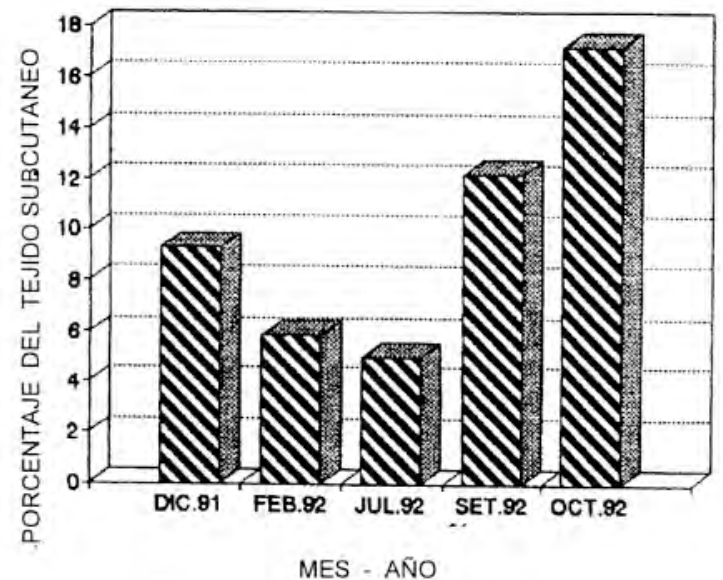

VI. BIBLIOGRAFIA

AMADORI, M. E. (1989). Acidos Grasos Esenciales Rev. Alimentos. Vol $14 \mathrm{~N}^{\circ} 2$. Santiago-Chile. Pág 76-79.

ARIMATSU, A. (1982). Métodos Quimicos de Análisis. Agencia de Cooperación Internacional de Japón. ITP-Callao.

BARRIOS, F.M. et. al. (1982). Factibilidad de Aprovechamiento de carne y grasa del lobo marino (Otaria flavescens). Rev. Alimentos Vol $7 \mathrm{~N}^{\circ} 1$. SantiagoChile. Pág. 9-13.

BAYLE, E.A. (1951). Aceites y Grasas Industriales. Ed. Reverté. Pag 147-152.

BERNARDINI, E. (1986). Tecnología de Aceites y Grasas. Edit. Alhambra. Pag 236, 258-261.

BJERREGAARD, B. (1992). Sroke in Greeland fathy acids and healtch. Omega-3 News. Vol VII N $N^{0}$. Boston-USA.

BRAUN, K. (1959). Grasas y Aceites. Manuales Técnicos Labor. Edit. Labor S.A.-Barcelona.

BUDSLAWSKY, J. y DRAKENT, Z. (1973). Metody Analizy Zywonosci. Wydawnictiva. Naukowo Techniczne, Wan.

CACERES P. (1991). Aceite de lobo marino (Otaria flavescens). Escrito propagandistico. Santiago-Chile.

CALIZAYA, H.I. (1988). Caracterización y refinación del aceite de sardina. Tesis Ing. Pesquero- UNJBG-Tacna.

CHEFTEL C., J. (1983). Introducción a la bioquímica y Tecnología de los alimentos. Edit. Acribia-ZaragozaEspaña.

COLOM V., R. (1956). Aceites y Grasas. Edit. Tip.Casals Barcelona-España.
GRÁFICO $N^{\circ}$ 5: Porcentaje de aceite obtenido del tejido subcutáneo en diferentes períodos de caza.

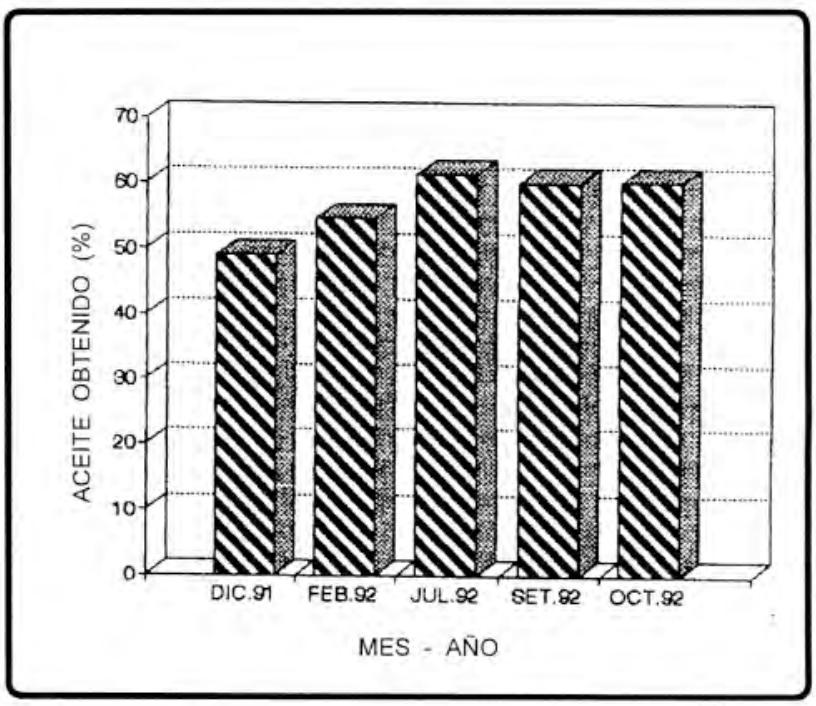

DASSOW J. A. (1959). Utilization of sea lions in Alaska U.S. Department of the interior fish and wildlife service. Sep. $N^{\circ} 425$, January.

DOLECEK T.A. (1992). An on going Evaluation of dietary polyinsaturated fatty acis and mortality in the multiple Risk factor intervention Trial. (MRFIT) Omega 3 Nws Vol. VII N 4 . Boston Ma.-USA.

HART y FISHER. (1984). Análisis moderno de alimentos. España.

NAKAMURA K. (1983). Acidos grasos poliinsaturados del aceite de pescado. Agencia de Cooperación Internacional de Japón- ITP, Callao.

LADONSKI (1986). Podstawowe metody analityczne produktow Varsovia-Polonia.

LEAF A. (1991). Fish oil suplements and antioxidants. Omega 3 News. Vol VI N ${ }^{0} 1$ Boston-Ma.- USA.

LEAF ALEXANDER. (1993). Omega 3 PUFA, an up daate: $1986-$ 1993. Omega 3 News. Department of preventive Medicine Massachusets General Hospital. Vol VIII Bostón - USA.

LEVANDER O. A. y AGER A. L. (1992). Omega 3 Fatty Acids oxidative stress and malaria. Omega 3 News $\mathrm{Vol}$ VII N'2, Boston - USA.

MAIER, H.G. (1978). Métodos modernos y análisis de alimentos VII. España Pag 129-131.

MARKOVITS A et al. (1989). Microalgas como alimento humano potencial y evaluación química y biológica de Phaeodactylum tricornutum. Rev. Alimentos Vol 14 $N^{0} 1$, Santiago-Chile. 
MASSON L. et al. (1988). Análisis y composición del extracto lipídico de las gónadas del erizo de mar (Loxechinus albus). Rev. Alimentos $\mathrm{Vol} 13 \mathrm{~N}^{\circ} 4$, Santiago-Chile Pag 35-38.

MACLENNAN D. y PETER L. (1993). Omega 3 Fatty acid effects on cardiac arrhytmias. Omega-3 News Vol VIII, Boston- USA.

MEHLENBACHER V. (1979). Aceites y Grasas. Análisis Enciclopedia de la Quimica Industrial. Edit. Urmo, S.A., España.

MÉTCALFE et al. (1966). Preparation of the fatty acids methyl esters. J. Anal. Chem. 38,514 New York-USA.

MÚRRAY R. y MAYES P. A. (1988). Bioquimica Harper. Edit. Manual Moderno, S. A. de C. V.

NAVARRETE et al. (1989). Acidos grasos y aminoácidos esenciales de la microalga Nannochloropsis sp. Revista Alimentos Vol $14 \mathrm{~N}^{\circ} 2$, Santiago-Chile.

PAZOS M. et al. (1990). Contenido y composición de los lípidos en las conservas del pescado del Perú. Boletín de Investigación Vol $3 \mathrm{~N}^{0} 1$ ITP, Callao-Perú.

PODESZENSK Z. y STODOLNIK, L. (1980). Cwiczenia Technologi Zabezpiecz nia Sorowcow Rybnych, Szczecin. Polonia.

SHERON R., L. y VALENCIA M., Q. (1993). Determinación de la composición física del lobo marino. Rev. Nueva Imagen. UNJBG. Tacna-Perú.

SPRECHER, H. (1992). A reevaluation of the pathway for the biosynthesis of $4,7,10,13,16,19$ Docosahexaenoic Acid. Omega-3 News Vol VII N ${ }^{\circ} 3$, Boston-USA.

STANSBY, M.E. (1967). Fish oil (their chemistry, Technology, Stability. Nutritional propierties and uses. The AVI Publishing Company, Inc. Pag 11-12, 397.

STUART M., B. (1980). Possible future pharmaceutical uses of fish oil. Twentisth Annual Conference International of fish MEAL Manufacturers- USA.

TOVAR, H. y FUENTES, H. (1984). Magnitud poblacional de lobos marinos en el litoral peruano en marzo de 1984. Informe $N^{\circ} 66$. IMARPE, Callao.

VALENCIA Q. et al. (1992). Proyecto sobre aprovechamiento integral de lobo marino. UNJBG-FAIP-MIPE, Tacna.

ZAITSEV V. et al. (1969). Fish curing and processing. Ed. Mir Publishers - Moscow.

ZALDIVAR J. (1989). Uso alternativo del aceite de pescado. Proyecto desarrollado por Universidad de Chile CORPESCA S.A.

ZALDIVAR L. JAVIER. (1992). Positivas proyecciones para los aceites de pescado. Rev. Chile Pesquero $N^{\circ} 70$. JunJul., Santiago-Chile. Pag 35-38

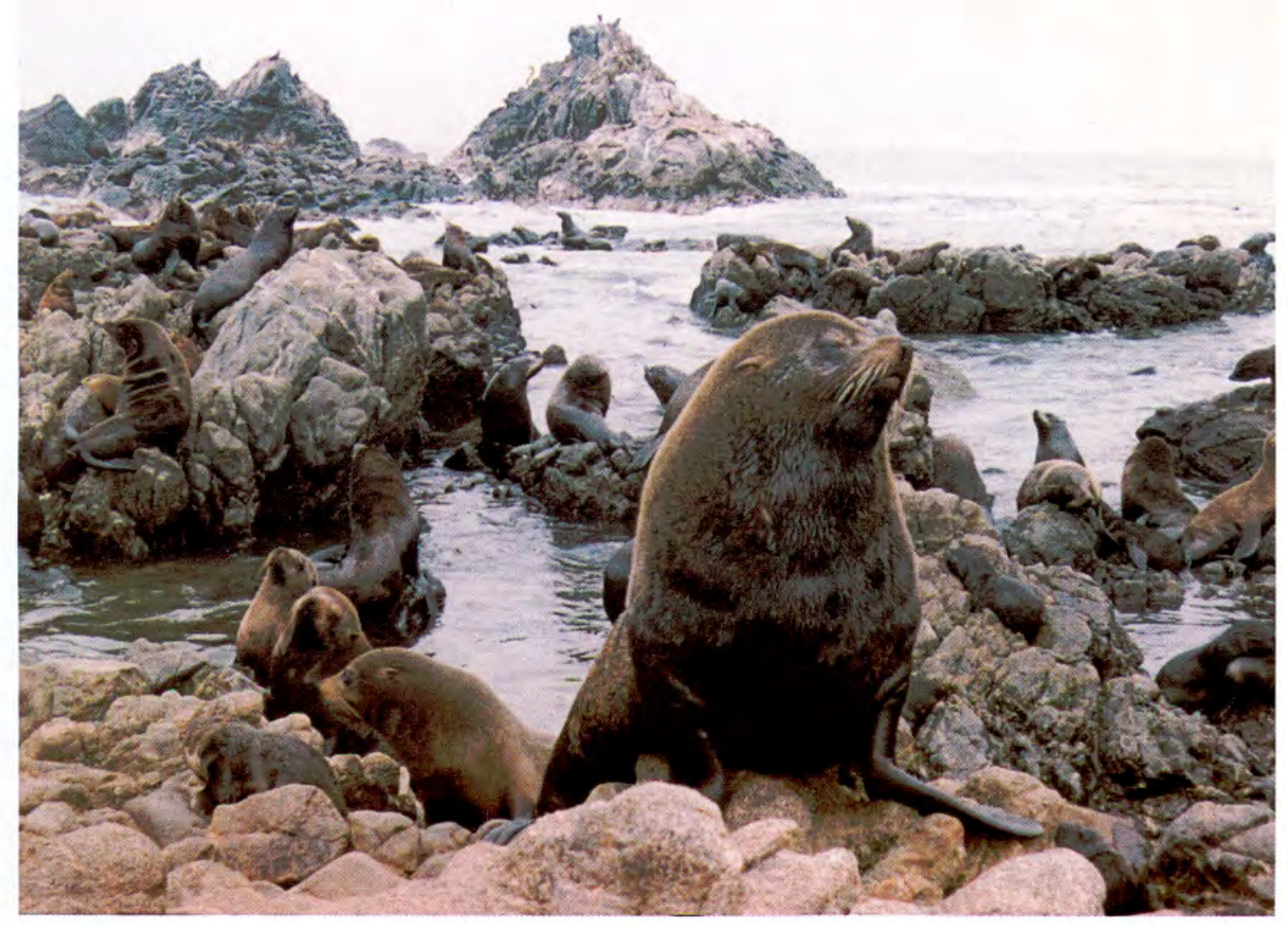

Colonias de lobos marinos en Punta de Coles - Ilo. 